\title{
УЧЕТ ЕSG ФАКТОРОВ ПРИ ФОРМИРОВАНИИ ИНВЕСТИЦИОННОГО ПОРТФЕЛЯ
}

\section{ENVIRONMENTAL, SOCIAL, AND GOVERNANCE FACTORS AS A PART OF THE INVESTMENT STRATEGY}

\section{A. Barysheva}

Summary. The paper provides an overview of methods for integrating environmental, social, and corporate governance (ESG) factors into the modern portfolio theory. According to the Global Alliance for Sustainable Investment (Global Sustainable Investment Alliance), the inclusion of ESG factors in the investment portfolio is the second most popular alternative investment strategy since 2018 [11, p. 10]. In the paper, we consider the main principles of ESG integration into an investment strategy, methods of obtaining ESG data, and show how to include ESG factors into the classic optimal Markowitz portfolio.

Keywords: Markowitz portfolio, environmental, social and governance factors, ESG, portfolio investment, alternative investment strategies.

\author{
Барышева Александра Евгеньевна \\ Программист-аналитик, ООО «ЭКО - ТОМСК» \\ (ТомсK) \\ alexandramelnikk@mail.ru
}

Аннотация. В настоящей работе представлен обзор методов интеграции факторов экологического, социального и корпоративного управления (ESG) в структуру современной портфельной теории. Согласно Глобальному Альянсу за Устойчивые Инвестиции (Global Sustainable Investment Alliance), включение ESG факторов в инвестиционный портфель является второй по популярности альтернативной инвестиционной стратегией с 2018 года [11, с. 10]. В рамках работы рассмотрены основные принципы интеграции ESG в инвестиционную стратегию, способы получения данных ESG, а также на примере была показана интеграция ESG в классический оптимальный портфель Марковица.

Ключевые слова: портфель Марковица, факторы экологического, социального и корпоративного управления, ESG, портфельное инвестирование, альтернативные инвестиционные стратегии.

годов появились инвестиционные стратегии, которые использовали нетрадиционные факторы как часть процесса принятия решений. Наиболее популярными среди них являются стратегии, включающие:

- социально-ответственное инвестирование (SRI для краткости);

- учет факторов экологического, социального и корпоративного управления (далее ESG факторы);

- корпоративная социальная ответственность (CSR для краткости) [10].

По данным Глобального Альянса Устойчивых Инвестиций (GSIA для краткости), в 2018 году такие альтернативные инвестиционные стратегии составляли 30,7 триллионов долларов США на пяти основных рынках в начале 2018 года (рост на 34\% за два года), как показано в таблице 1.

\section{1. История появления ESG Факторов}

Термин ESG происходит от концепции Triple Bottom Line (TBL), также известной как Люди, Планета и Прибыль (или просто ЗР), введенной Элкингтоном в его книге [8]. Он определил несколько нефинансовых факторов, которые, по его мнению, следует учитывать при 
Таблица 1. Альтернативные инвестиции по данным GSIA, 2016-2018

\begin{tabular}{|c|c|c|}
\hline Регион & 2016, млрд. \$ & 2018, млрд. \$ \\
\hline Европа & 12040 & 14075 \\
\hline США & 8723 & 11995 \\
\hline Япония & 474 & 2180 \\
\hline Канада & 1086 & 1699 \\
\hline Австралия & 516 & 734 \\
\hline Всего & 22890 & 30683 \\
\hline
\end{tabular}

оценке долгосрочных перспектив бизнеса. Эти идентифицированные элементы включают три измерения:

1. Социальный (люди): измеряет степень социальной ответственности бизнеса.

2. Экологический (планета): измеряет влияние деловой активности на окружающую среду.

3. Финансовый (прибыль): идентифицируется из отчета о прибылях и убытках (отчет о прибылях и убытках).

Появление данного подхода стимулировалось разочарованием традиционными (то есть, сфокусированными на финансах) показателями эффективности бизнеса, которые, как правило, выделяли прибыль ключевым показателем.

\section{2. ВнеАрение ESG фактOрOв в инвестиционную стратегию}

Внедрение ESG факторов подразумевает инвестиционную стратегию, которая объединяет существенные (т.е. влияющие на финансовые показатели) факторы ESG с традиционными финансовыми показателями для анализа компаний. Экологические факторы включают в себя отношение компании к природной среде, например, то, как компания решает такие проблемы, как загрязнение, использование энергии или использование воды. Социальные факторы нацелены на трудовые отношения, включая обращение с работниками, условия для работников и вознаграждение работников. Социальные факторы также включают в себя то, как компания взаимодействует с сообществами, в которых она работает. Факторы управления сконцентрированы на том, как компания управляет собой, включая вознаграждение руководителей, внутренний контроль, аудит и прозрачность для акционеров и общественности.

Финансовые аналитики используют внедрение ESG факторов для улучшения выбора акций, поскольку факторы ESG могут помочь определить потенциальные возможности и риски компании. Интеграция ESG позволяет учесть информацию, имеющую отношение к сильным и слабым сторонам компании, и, следова- тельно, потенциально может привести к принятию более эффективных инвестиционных решений. Например, в [2] автор описывает интеграцию ESG как «золотой стандарт» ответственных инвестиций из-за его эффективности в сочетании финансовой, экологической и социальной выгоды. Поскольку все больше доказательств показывает, что учет факторов ESG может улучшить доходность портфеля с поправкой на риск, все больше финансовых аналитиков используют некоторую форму интеграции ESG. Многочисленные исследования, сравнивающие фонды SRI с фондами не-SRI или с рыночными ориентирами, в целом не выявили различий в результатах между фондами, определенным в какой-то степени социально ответственными, и фондами без такого назначения. Исследования, которые смотрят на интеграцию ESG более детально, показывают большую вероятность улучшения результатов портфеля при учете ESG факторов.

В настоящее время нет единого мнения ни в академических кругах, ни в отрасли относительно точного определения критериев ESG [31]. Тем не менее, среди участников отрасли были многочисленные попытки по их стандартизации. Во-первых, существует обширный каталог, опубликованный Европейской федерацией обществ финансовых аналитиков (EFFAS для краткости), В котором указаны ключевые показатели эффективности (KPI) для вопросов ESG. Рассматриваемые KPI делятся на общие и отраслевые. Помимо уже упомянутой ESG, каталог включает в себя так называемую долгосрочную жизнеспособность, которая предназначена для охвата тех KPI, которые отражают концепцию устойчивости рынков капитала. Точнее говоря, он представляет собой способность компании получать долгосрочную прибыль, не жертвуя активами, навыками или ресурсами из-за краткосрочной эксплуатации [7]. Во-вторых, существуют Принципы ответственного инвестирования ООН (сокращенно, UNPRI), в которых, однако, подчеркивается, что список пунктов ESG нежелателен из-за динамического характера требований ESG к любому участнику. Вместо этого в UNPRI приводятся некоторые общие рекомендации относительно того, что следует включать в ESG, как показано в таблице 2. 
Таблица 2. Классификация факторов ESG согласно UNPRI.

\begin{tabular}{|l|l|l|}
\hline E (экологические) & S(социальные) & G (управленческие) \\
\hline Изменение климата, & Человеческие права, & Взятки и коррупция, \\
Истощение ресурсов, & Современное рабство, & Своевременная оплата труда, \\
Отходы, & Детский труд, & Прозрачная структра управления, \\
Выброс газов, & Условия труда, & Налоговая стратегия, \\
Загрязнение окр.среды. & Птноение работодателя & Политическое лоббирование \\
\hline
\end{tabular}

Несмотря на соглашение по определенным широким определениям, таким как вышеупомянутые экологические, социальные и управленческие вопросы, есть очевидные причины, по которым трудно найти консенсус по сути ESG. Концепции ESG отличаются не только от сектора к сектору, но также сильно зависят от точки зрения заинтересованных сторон. Нормативные требования, инвесторы, компании или неправительственные организации, вероятно, будут отличаться в своих представлениях о том, какие ESG факторы следует включать.

\section{3. По $\triangle$ хо $\mathrm{k}$ к внеАрению ESG фактOрОв в инвестиционную стратегию}

Наиболее популярной инвестиционной стратегией ESG является исключение или отрицательный скрининг. Стратегия в основном избегает компаний или секторов, которые не соответствуют этическим или ESG-критериям, определенным инвестором. В зависимости от инвестора это может быть мотивировано соображениями управления рисками, требованиями об ответственности, а также религиозными принципами. Такая стратегия может быть добровольно выбрана или применена законом. В целом критерии исключения может быть применена за нарушение прав человека, плохие условия труда, экологическую беспечность, обороны, испытания на животных, алкоголь, табак, азартные игры, порнография и т.д. На практике часто определяют пороговое значение, например, в процентах от максимальной прибыли компании, получаемой в результате несоответствующей деловой активности. При выполнении вышеупомянутого предварительного исключения применяется финансовый и количественный отбор. Другая стратегия, похожая на исключение,- это скрининг на основе определенных стандартов. Здесь применяется набор стандартов, которые обычно предопределяются международной организацией, такой как Организация экономического сотрудничества и развития (ОЭСР) или Глобальное воздействие ООН (UNGI). Инвесторы обычно реагируют на несоблюдение этих стандартов, во-первых, проводя более глубокий анализ и, во-вторых, либо исключая соответствующие инвестиции из портфеля, либо взаимодействуя с соответствующей компанией.
Помимо двух отрицательных стратегий фильтрации, существует также положительная стратегия, называемая лучшей в своем классе. После этого положительного скрининга слабые исполнители ESG «наказываются» или исключаются, а сильные получают вознаграждение или включаются. На практике при выборе портфеля учитывается только определенный верхний перцентиль в отношении критериев ESG в секторе. Таким образом, сужается не только вселенная для инвестиций, но и относительное распределение в портфеле может зависеть от факторов ESG, а не только критериев финансового вклада.

Последней стратегией, рассматриваемой в этой статье, является интеграция ESG, которая определяется как явное включение рисков и возможностей ESG в традиционный финансовый анализ. Это включение охватывает использование как качественной, так и количественной информации ESG в инвестиционном процессе и направлено на повышение эффективности принятия инвестиционных решений. Интеграция может использоваться на уровне портфеля, акций, эмитента или уровня инвестиций. Акцент делается на интеграцию критериев ESG в традиционный финансовый анализ ценных бумаг и компаний, помимо риска и доходности. В этом также заключается один из потенциальных барьеров для управляющих активами при реализации интеграции: риск и доход являются объективно измеримыми критериями, в то время как оценки ESG оспариваются с точки зрения сопоставимости и прозрачности.

\section{4. $\triangle$ анные $\triangle \wedge$ я ошенки ESG}

Bсе инвестиционные стратегии ESG, рассмотренные в предыдущем разделе, основаны на данных ESG компании. Следуя [15, с. 26-27], растущий интерес к инвестиционным стратегиям ESG в сочетании с уже упоминавшимся отсутствием стандартизации привел к формированию нескольких индексов и рейтинговых агентств (обратите внимание, что первые рейтинги ESG появились в 1980-х годах как услуга для инвесторов по проверке компаний, не только по финансовым характеристикам, а также по характеристикам, связанным с социальными и экологическими показателями [1, с. 5]). Все они предоставляют определенный рейтинг 
ESG компании (например, число от 0 до 100). Анализируя рейтинговый рынок ESG, французская рейтинговая компания Novethic обнаружила, что на рынке наблюдается консолидация агентств, но работа становится все более разнообразной. Обычно рынок данных ESG можно разделить на две части: компании, предоставляющие рейтинги, и компании, предоставляющие необработанные данные (которые могут использоваться инвесторами для создания собственных рейтингов). Компании, которые предоставляют рейтинги, обычно количественно оценивают необработанные данные для измеримых KPI или рейтингов. Рейтинговые агентства обычно объединяют политики ESG компании и реализацию, исполнение и отчетность этих политик. Рейтинговые агентства ESG, упомянутые, например, в $[13,15,31]$, включают Индекс устойчивых инвестиций Folksam, индекс ESG STOXX и Thomson Reuters. Thomson Reuters признает, что сегодня многие инвесторы хотели бы инвестировать ответственно. Таким образом, он направлен на поддержку желания путем предоставления этих баллов ESG. Компания заявляет, что «результаты Thomson Reuters ESG Scores предназначены для прозрачного и объективного измерения относительной эффективности, приверженности и эффективности ESG компании по 10 основным темам (выбросы, инновации в области экологических продуктов, права человека, акционеры и т.д.) на основе данных, представленных компанией» [23, с. 3]. Баллы доступны в форме простого перцентиля: как в процентах, так и в виде буквенной оценки от D- до A +. Эти оценки сравниваются с отраслевой группой бизнес-классификаций Thomson Reuters. Оценки ESG являются улучшением базы данных «Asset4» [23, с. 3]. Сопоставимость улучшается за счет введения контрольных показателей на уровне отрасли и страны на уровне оценки данных. Веса категорий, управляемые данными, добавляются для того, чтобы отразить доступность данных в каждой категории, таким образом, поддерживая дифференциацию между компаниями $[23$, с. 3].

С точки зрения Thomson Reuters экологические ESG аспекты заключаются в следующем:

1. Использование ресурсов: это включает способность компании использовать как можно меньше с точки зрения материалов, воды и энергии. Поиск более экологичных подходов рассматривается как преимущество.

2. Выбросы: измеряет эффективность и приверженность компании сокращению выбросов при производстве и других производственных процессах.

3. Инновации: отражает способность компании внедрять инновации таким образом, чтобы снизить экологические издержки и бремя для своей клиентской базы, что можно сделать путем создания новых рыночных возможностей, экологических технологий или разработки экологически чистых продуктов.

Социальные аспекты включают в себя следующее:

1. Рабочая сила: включает в себя эффективность в повышении удовлетворенности работой, безопасное и здоровое рабочее место, открытость для разнообразия и поддержку равных возможностей, а также возможностей для развития.

2. Права человека: просто исследует, насколько эффективно компания придерживается основных конвенций по правам человека.

3. Сообщество: измеряет приверженность компании стремлению быть хорошим корпоративным гражданином, защите общественного здоровья и ведению этического бизнеса.

4. Ответственность за продукцию: измеряет способность производить качественные товары и услуги, сохраняя при этом здоровье, безопасность, целостность и конфиденциальность данных клиента.

Наконец, аспекты управления состоят из:

1. Управление: измеряет приверженность и эффективность в соответствии с передовыми принципами корпоративного управления.

2. Акционеры: меры по равному обращению с акционерами и использованию средств защиты от поглощения.

3. Стратегия: отражает усилия компании по интеграции экономических, социальных и экологических аспектов в повседневные процессы.

Кроме того, Thomson Reuters заявляет, что все вышеперечисленные десять категорий имеют бальную структуру. Например, подсчет рабочей силы состоит из 29 различных показателей. Баллы по стратегии в области прав человека и стратегии имеют наименьшее количество отдельных подкатегорий из 8. Всего имеется 178 показателей ESG, которые собираются и анализируются до последующего формирования балла ESG $[23$, с. 8]. Данные собираются из шести основных источников: годовых отчетов, веб-сайтов компаний, веб-сайтов НПО, фондовых бирж, отчетов по КСО и новостных источников. Для достижения наилучшего качества данных используются как человеческие, так и алгоритмические процессы.

\section{5. ВнеАрение ESG факторов в к^ассическую портфельную теорию}

Напомним, что с учетом $n$ активов соответствующая доходность портфеля $R_{P}$ может быть рассчитана как: 
Таблица 3. Доходность индексов на 31 января 2020 г.

\begin{tabular}{|c|c|c|c|c|c|c|c|}
\hline & \multirow{2}{*}{$1 \mathrm{M}$} & \multirow{2}{*}{$3 M$} & \multirow{2}{*}{$1 Y$} & \multicolumn{4}{|c|}{ \% годовых } \\
\hline & & & & $3 Y$ & $5 Y$ & $10 Y$ & C2007 \\
\hline MSCI World ESG Leaders & 0,02 & 5,78 & 20,23 & 12,54 & 9,69 & 10,39 & 5,77 \\
\hline MSCI World & $-0,58$ & 5,34 & 18,4 & 12,08 & 9,62 & 10,48 & 5,69 \\
\hline
\end{tabular}

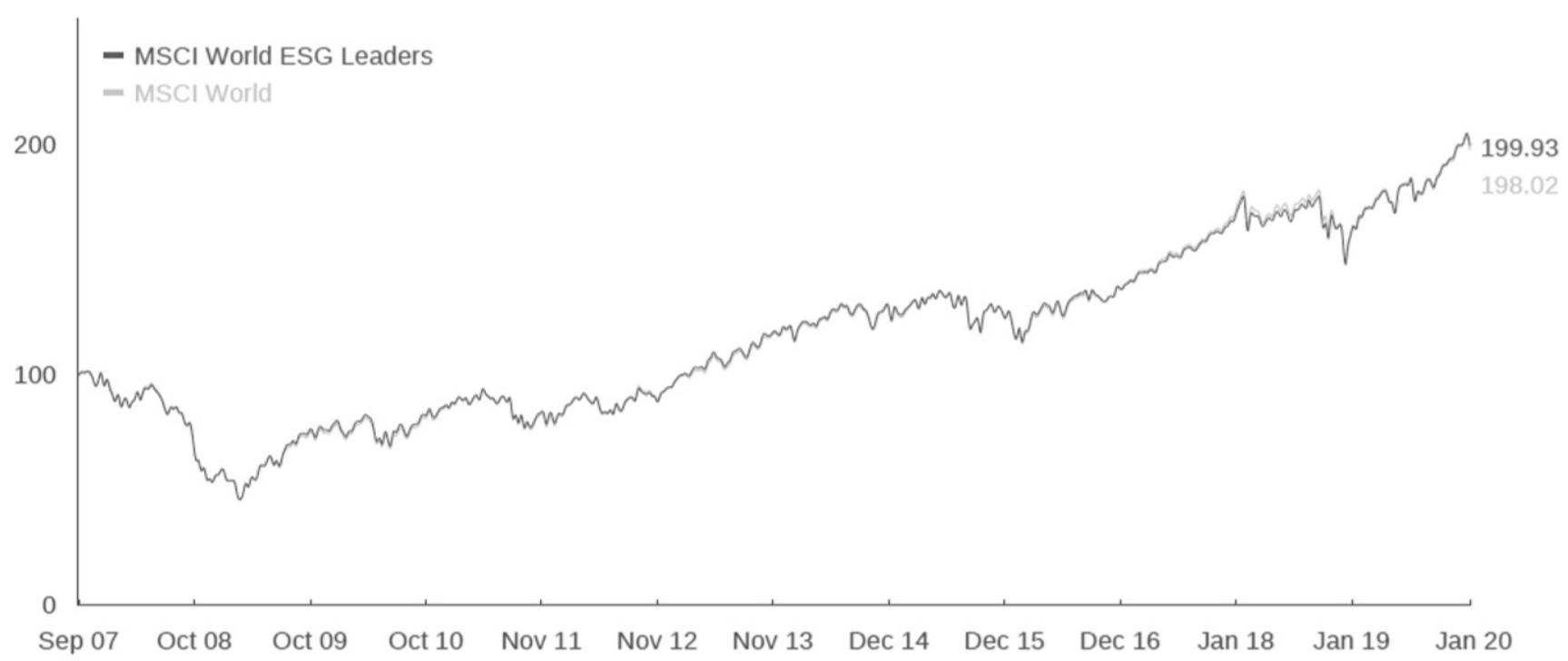

Рис. 1. Совокупный показатель эффективности - валовый доход (долл. США) (сентябрь 2007 г.— январь 2020 г.)

$E\left(R_{p}\right)=\sum_{i=1}^{n} x_{i} E\left(R_{i}\right)=\sum_{i=1}^{n} x_{i} \mu_{i}=x^{T} \mu$ феле.

где $R_{i}$ - доходность актива $i, x_{i}$ - вес актива $i$ в порт-

Риск портфеля записывается в виде:

$$
\operatorname{Var}\left(R_{P}\right)=x^{T} \sum x, \Sigma=\left(\begin{array}{ccc}
\sigma_{11} & \cdots & \sigma_{1 n} \\
\vdots & \ddots & \vdots \\
\sigma_{n 1} & \cdots & \sigma_{n n}
\end{array}\right)
$$

где $\Sigma$ есть матрица ковариаций активов портфеля, $\sigma_{i, j}$ отображает ковариацию между активами $i, j$. Тогда оптимизационная задача Марковица может быть записана в следующем виде:

$$
\left\{\begin{array}{c}
x^{T} \sum x \rightarrow \min \\
x^{T} \mu \geq \lambda \\
x^{T} E S G \geq E S G_{\text {tagret }} \\
x^{T} 1=1
\end{array}\right.
$$

\section{6. ВАияние внеАрения ESG

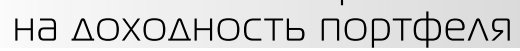

B настоящее время нет единого мнения по вопросу влияния факторов ESG, включенных в портфель на его доходность. Стратегии устойчивого инвестирования (и, следовательно, их варианты с ESG), как правило, ориентированы на долгосрочное инвестирование, что подпитывает заблуждение, что такие стратегии менее эффективны, чем классические. Тем не менее, рассмотрим рисунок 1, на котором показаны показатели MSCI World ESG Leaders и индексы MSCI World (USD) за последние 13 лет.

Кроме того, в таблице 3 представлены показатели обоих индексов в виде доходности за несколько периодов времени.

Как видно из таблицы 3 (и из рисунка 3), MSCI World ESG Leaders превосходит MSCI World за все рассматриваемые периоды времени, исключая 10-летний период, который можно рассматривать как выброс. Наиболь- 
шее различие наблюдается в течение 1 года, когда MSCI World ESG Leaders превосходит MSCI World почти на 2\%, при этом другие различия не совсем значительны.

\section{Зак^ючение}

В данном исследовании представлен краткий обзор одного из вариантов устойчивого инвестирования, а именно интеграции ESG в Классическую портфельную теорию Марковица. Как указано в [10, с. 801], «Интеграция ESG - это не просто новый термин для социальных инвестиций, которые описывают стратегию, фокусирующуюся на экологических или социальных воздействиях без учета финансовых факторов. Скорее, интеграция
ESG объединяет традиционные финансовые показатели с информацией, касающейся экологических, социальных или управленческих поведений компании или рисков для улучшения анализа потенциала компании как инвестиции». Согласно [11, с. 10], интеграция ESG является второй по популярности среди стратегий устойчивого инвестирования, в которой рост активов составил 69\% за период 2016-2018 гг. Несмотря на очевидный рост интереса к альтернативным инвестиционным стратегиям, основанным на «социальной ответственности», уже имеющаяся практическая реализация еще не созрела по двум основным причинам:

1. Отсутствие стандартизации к оценке ESG;

2. Отсутствие у инвесторов веры в эффективность;

\section{ЛИТЕРАТУРА}

1. F. Berg, J.F. Koelbel, and R. Rigobon, Aggregate Confusion: The Divergence of ESG Ratings, MIT Sloan School of Management, MIT Sloan School Working Paper 5822-19, August 2019.

2. M. Cappucci, The ESG integration paradox, Journal of Applied Corporate Finance 30 (2018), no. 2, 22-28.

3. V. DeMiguel, L. Garlappi, and R. Uppal, Optimal versus naive diversification: How inefficient is the $1 / \mathrm{N}$ portfolio strategy?, The review of Financial studies 22 (2009), no. 5, 1915-1953.

4. A. Descl'ee, L. Dynkin, J. Hyman, and S. Polbennikov, Sustainable investing and bond returns. Research study into the impact of ESG on credit portfolio performance, Impact Series 01, Barclays Bank PLC, 2016.

5. B. Drut, Social responsibility and mean-variance portfolio selection, CEB Working Paper 10/002, Universit'e Libre de Bruxelles — Solvay Brusells School of Economics and Management, Centre Emile Benrheim, January 2010.

6. DWS, Digging Deeper into the ESG Corporate Financial Performance Relationship, DWS Investment GmbH, Global Research Institute, September 2018, available online at https://download.dws.com/download?elib-assetguid=714aed4c2e83471787d1 ca0f1b559006.

7. EFFAS, KPIs for ESG. A Guideline for the Integration of ESG into Financial Analysis and Corporate Valuation, EFFAS The European Federation of Financial Analysts Societies, Version 1.2, 2009, available online at http://ec.europa.eu/DocsRoom/documents/1547/attachments/1/translations/en/renditions/pdf.

8. J. Elkington, Cannibals with Forks: The Triple Bottom Line of 21st Century Business, Capstone Publishing Limited, Oxford Center for Innovation, 1997.

9. EU, Sustainable finance: Council agrees position on a unified EU classification system, Council of the EU, Press Release 608/19, 25/09/2019, September 2019, available online at https://www.consilium.europa.eu/en/press/press-releases/ 2019/09/25/sustainable-finance-council-agrees-position-on-a-unified-euclassification-system/pdf.

10. S.N. Gary, Best interests in the long term: fiduciary duties and ESG integration, University of Colorado Law Review 90 (2019), 731-801.

11. GSIA, Global Sustainable Investment Review 2018, Global Sustainable Investment Alliance, 2019, available online at http://www.gsi-alliance.org/wp-content/ uploads/2019/03/GSIR_Review2018.3.28.pdf.

12. J.C. Hull, Options, Futures, and Other Derivatives, 10th ed., Pearson, NY, 2018.

13. R. Jakobsson and L. Lundberg, The Effect of ESG Performance on Share Price Volatility, Degree Project, Department of Business Administration, Ume a School of Business, Economics and Statistics, Ume a University, 2018.

14. J. Lintner, The valuation of risk assets and the selection of risky investments in stock portfolios and capital budgets, The Review of Economics and Statistics 47 (1965), no. 1, 13-37.

15. E. Lundstr" om and C. Svensson, Including ESG concerns in the portfolio selection process: an MCDM approach, Degree Project in Applied Mathematics and Industrial Economics, KTH Royal Institute of Technology, SCI School of Engineering Sciences, Stockholm, Sweden, 2014.

16. B. Marigo, Socially Responsible Investment: integrating financial and non-financial criteria into a portfolio selection model, Master's thesis, Universit’ a Ca'Foscari Venezia, 2016.

17. H. Markowitz, Portfolio selection, The Journal of Finance 7 (1952), no. 1, 77-91.

18. H. Markowitz, Portfolio Selection: Efficient Diversification of Investments, John Wiley, New York, 1959.

19. H. Markowitz, The early history of portfolio theory: 1600-1960, Financial Analysts Journal 55 (1999), no. 4, 5-16.

20. J. Mossin, Equilibrium in a capital asset market, Econometrica 34 (1966), no. 4, 768-783.

21. MSCI, MSCI World ESG Leaders Index (USD), MSCI Inc., January 2020, available online at https://www.msci.com/ documents/10199/db88cb95-3bf3-424cb776-bfdcca67d460.

22. I. Oikonomou, E. Platanakis, and C. Sutcliffe, Socially responsible investment portfolios: Does the optimization process matter?, The British Accounting Review 50 (2018), no. 4, 379-401.1. 14 
23. Refinitiv, Environmental, Social and Governance (ESG) Scores from Refinitiv, Refinitiv, June 2019, available online at https://www.refinitiv.com/content/dam/ marketing/en_us/documents/methodology/esg-scores-methodology.pdf.

24. G. Serafeim, J. Huang, J. Linder, P. Faul, and J. Streur, The Financial and Societal Benefits of ESG Integration: Focus on Materiality, The Calvert-Serafeim Series, June 2016, available online at https://www.hvst.com/posts/the-financialand-societal-benefits-of-esg-integration-focus-on-materiality-X34TPKjd.

25. J. Serafeim, The role of the Corporation in Society: An Alternative View and Opportunities for Future Research, Working Paper 14-110, Harvard Business School, May 2014.

26. W.F. Sharpe, Capital asset prices: A theory of market equilibrium under conditions of risk, The Journal of Finance 19 (1964), no. 3, 425-442.

27. M.C. Steinbach, Markowitz revisited: mean-variance models in financial portfolio analysis, SIAM review 43 (2001), no. 1, 31-85.

28. J.L. Treynor, Market value, time, and risk, unpublished manuscript, 1961.

29. J.L. Treynor, Toward a theory of market value of risky assets, unpublished manuscript, 1962.

30. UNPRI, What is reponsible investment?, United Nations Principles for Responsible Investment, 2019, available online at https://www.unpri.org/ download?ac=6998. [31] P. Zuber, Risk, Return, Responsibility — Inclusion of ESG Criteria in a Portfolio Optimization Framework, Master's thesis, Center for Microfinance, Department of Banking and Finance, University of Zurich, January 2017.

31. Барышева А.Е. Актуальные проблемы портфельного инвестирования/ Экономика и предпринимательство. 2020.— № 10. С.— $887-882$.

( Б) Барышева Александра Евгеньевна ( alexandramelnikk@mail.ru ).

Журнал «Современная наука: актуальные проблемы теории и практики»

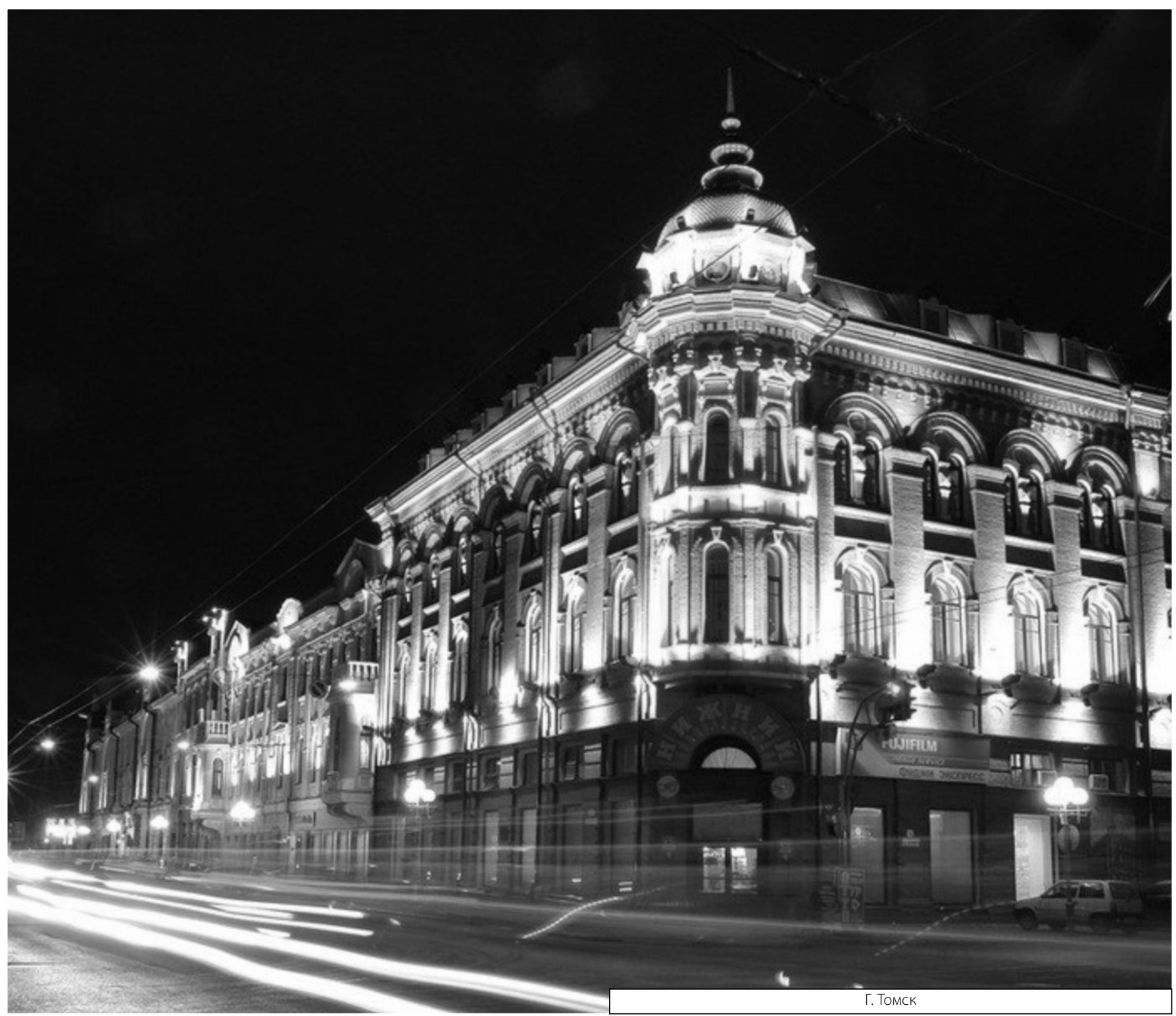

